\title{
Elevated osteogenic potential of stem cells from inflammatory dental pulp tissues by Wnt4 overexpression for treating bone defect in rats
}

\author{
Tianyu Zhong ${ }^{1,2,3}$, Yunan Gao ${ }^{1,2}$, Hu Qiao ${ }^{1,2}$, Hong Zhou ${ }^{1,2}$, Yong Liu ${ }^{1,3}$ \\ ${ }^{1}$ Key Laboratory of Shaanxi Province for Craniofacial Precision Medicine Research, Xi'an Jiaotong University, Xi'an, China; ${ }^{2}$ Department of \\ Orthodontics, The Affiliated Stomatological Hospital of Xi'an Jiaotong University, Xi'an, China; ${ }^{3}$ Institute of Neurobiology, Xi'an Jiaotong \\ University Health Science Center, Xi'an, China \\ Contributions: (I) Conception and design: T Zhong, Y Liu, H Zhou; (II) Administrative support: Y Liu, H Zhou; (III) Provision of study materials or \\ patients: T Zhong, Y Gao, H Qiao; (IV) Collection and assembly of data: T Zhong; (V) Data analysis and interpretation: T Zhong; (VI) Manuscript \\ writing: All authors; (VII) Final approval of manuscript: All authors. \\ Correspondence to: Yong Liu. Institute of Neurobiology, Xi'an Jiaotong University Health Science Center, \#76 Yanta West Road, Xi'an, China. Email: \\ liuy5599@mail.xjtu.edu.cn; Hong Zhou. Department of Orthodontics, The Affiliated Stomatological Hospital of Xi'an Jiaotong University, Xi'an, \\ China. Email: zhouhong@mail.xjtu.edu.cn.
}

Background: The osteogenic capacity of inflammatory dental pulp stem cells (DPSCs-IPs) is reported lower than that of normal dental pulp stem cells (DPSCs-NPs). Down-regulation of Wnt4 may be the key factor affecting the osteogenic ability of DPSCs-IPs. In order to prove that the restoration of Wnt4 expression could improve the osteogenic potential of DPSCs-IPs, Wnt4 overexpressed inflammatory dental pulp stem cells (Wnt4-DPSCs-IPs) were performed to reconstruct bone defects in rats.

Methods: Human DPSCs-IPs were cultured and transfected with Wnt4 overexpression lentiviral vector. Stem cell characterization was performed by flow cytometry and induction of multidirectional differentiation. Wnt4-DPSCs-IPs were loaded onto poly (hydroxybutyrate-co-valerate) (PHBV). The compounds were engrafted into artificially-created defect in alveolar bone. The effectiveness of Wnt4-DPSCs-IPs/PHBV in bone regeneration was assessed by micro-CT and immunohistochemical staining of osteocalcin, a representative osteogenic marker.

Results: Collecting data showed that Wnt4 overexpression didn't change stem cell characteristics of DPSCs-IPs. Wnt4-DPSCs-IPs retain osteogenic, adipogenic and chondrogenic differentiation abilities. Wnt4-DPSCs-IPs/PHBV were more effective than DPSCs-IPs/PHBV in repair of rat bone defects by 3 months' post-surgical reconstruction.

Conclusions: Restoration of Wnt4 expression could improve the osteogenic potential of DPSCs-IPs. Wnt4 restored DPSCs-IPs may be a feasible resource of seed cells for bone regeneration in future clinical application.

Keywords: Inflammatory dental pulp stem cells (DPSCs-IPs); Wnt4; bone regeneration

Submitted Feb 20, 2020. Accepted for publication Jun 18, 2020.

doi: $10.21037 /$ apm-20-460

View this article at: http://dx.doi.org/10.21037/apm-20-460

\section{Introduction}

Recently evidence indicated that inflammatory dental pulp tissues retained tissue regeneration potential by containing a certain proportion of mesenchymal stem cells, inflammatory dental pulp stem cells (DPSCs-IPs) $(1,2)$. However, the osteogenic potential was inhibited to some extent compared with normal dental pulp stem cells (DPSCs-NPs) $(3,4)$. DPSCs-NPs have been proved to be a good source of seed 
cells in tissue engineering (5-10). While the main challenge in clinical application is the availability, since patients must sacrifice dental pulp tissues in normal tooth for culturing DPSCs-NPs (11-13). However, there are numerous patients who have already had their dental pulp infected and the extracted pulp turned medical waste. If we can find a way to improve the osteogenic capacity of DPSCs-IPs, we can turn waste into treasure in the clinic.

Previous studies showed that the down-regulation of Wnt4 accounted for the impaired osteogenic potential of DPSCs under inflammatory conditions (14-16). Wnt signaling plays a key role in the osteogenic differentiation of mesenchymal stem cells (17). Studies have found that Wnt4 (a non-classical Wnt member) was crucial in promoting the osteogenic differentiation of MSCs isolated from craniofacial tissues (18). In our previous study, Wnt4 regulated C-Jun pathway was down regulated in inflammatory conditions (19). In addition, we also found that Wnt4 rescue promoted the osteogenic differentiation of DPSCs-IPs as well as the related gene expression. Our present study aims to figure out whether the restore of Wnt4 in DPSCs-IPs engrafted in poly (hydroxybutyrateco-valerate) (PHBV) has the developed ability of reconstructing bone defect in rats. We present the following article in accordance with the ARRIVE reporting checklist (available at http://dx.doi.org/10.21037/apm-20-460).

\section{Methods}

\section{Cell culture}

Dental pulp (normal and inflamed) were obtained from Stomatological Hospital, Xi'an Jiaotong University, and was approved by the Ethics Committee of Stomatological Hospital, College of Medicine, and Xi'an Jiaotong University. Extracted pulps were digested by $3 \mathrm{mg} / \mathrm{mL}$ dispase II (Roche, Germany) and $3 \mathrm{mg} / \mathrm{mL}$ type IV collagenase (STEMCELL Technologies, Canada). After $1 \mathrm{~h}$ digestion, cells were suspended in $10 \mathrm{~cm}$ dish with Dulbecco's-modified Eagle's media (DMEM) containing $10 \%$ fetal bovine serum (FBS) and $1 \%$ penicillin/ streptomycin (all from Gibco, USA). The full-length sequence of Wnt4 was amplified by PCR and connected to the lentiviral vector (Genechem, China). DPSCsIPs at passage 3 were collected to transduct recombinant lentiviral Wnt4. Stably over-expressing Wnt4 cell line was screened out.

\section{Flow cytometry for characterization of Wnt4-DPSCs-IPs}

Passage 1 of Wnt-DPSCs-IPs was collected to detect surface markers by flow cytometry. Primary antibodies used were: mouse monoclonal anti-human STRO-1, CD105 and CD45 and rabbit monoclonal anti-human CD34 and CD90 (all from Abcam, Cambridge, MA, USA).

\section{Multidirectional differentiation of Wnt4-DPSCs-IPs}

Osteogenic, adipogenic and chondrogenic differentiation induction were also performed to characterize Wnt4DPSCs-IPs. For osteogenic differentiation, cells were cultured with induction media (HUXDP-90021, Cyagen, China) for 14 days for Alizarin Red S (ARS) staining. For adipogenic differentiation, cells were cultured with induction media (HUXDP-90031, Cyagen, China) for 14 days for oil-red $\mathrm{O}$ staining. For chondrogenic differentiation, cells were cultured with induction media (HUXDP-90041, Cyagen, China) for 14 days for toluidine blue staining. For ARS staining, calcified nodules were evaluated, for oil-red $\mathrm{O}$ staining, red lipids were calculated, and for Toluidine Blue staining, blue-purple chondrocytes were counted.

\section{Preparation and evaluation of complex Wnt4-DPSCs-IPs/ PHBV}

One $\mathrm{cm}^{2}$ PHBV (thickness: $0.2 \mu \mathrm{m}$ ) was placed in one well in a 24-well plate, Wnt4-DPSCs-IPs were engrafted on PHBV. Culture medium was generally changed every 3 days. Fourteen days later, the complex was scraped for scanning electron microscope analysis.

\section{Cell proliferation and ALP assay}

Zero point three $\mathrm{cm}^{2}$ PHBV (thickness: $0.2 \mu \mathrm{m}$ ) was placed in one well in a 96-well plate, Wnt4-DPSCs-IPs were seeded at a density of $5 \times 10^{3}$ cells per well. Cells were cultured at $37^{\circ} \mathrm{C}$ for 1,3 , and 7 days. Cell proliferation was detected by MTT analysis. Cells cultured with osteogenic induction media for 14 days were used to detect ALP activity by alkaline phosphatase (ALP) detection kit (G5610, Solarbio, China) according to the product instructions. 


\section{Generation of a bone defects in rats}

Twenty 4-week-old male Sprague Dawley rats (weighing $200 \mathrm{~g}$ ) were used in the experiment. They were all treated in accordance with the guidelines of Xi'an Jiaotong University College of Medicine for Experimental Animals. Bone defect model was standardized built with a size of $2 \times 1 \times 1 \mathrm{~mm}$ at retromolar area in maxillary.

\section{In vivo transplantation and evaluations}

There are four treatment groups: (I) PHBV (n=5): treatment with only PHBV; (II) DPSCs-IPs/PHBV ( $\mathrm{n}=5$ ): DPSCsIPs engrafted on PHBV; (III) DPSCs-NPs/PHBV $(\mathrm{n}=5)$ : DPSCs-NPs engrafted on PHBV; (IV) Wnt4-DPSCs-IPs/ PHBV (n=5): Wnt4 overexpressed DPSCs-IPs engrafted on PHBV. Micro-CT was used to examine bone regeneration 3 months post-transplantation. Maxillary were fixed in 4\% paraformaldehyde. Tissues were scanned on a micro-CT system. The parameters are set as follows: $70 \mathrm{kV}, 114 \mu \mathrm{A}$, $12 \mu \mathrm{m}$ resolution. CTAn software was used to analysis.

\section{Immunobistochemistry}

Deparaffinize and rehydrate slides. Wash slides for 3 times each. Incubate with $0.1 \%$ Trypsin (T8150, Solarbio, China) at $37^{\circ} \mathrm{C}$ for $10 \mathrm{~min}$. Rinse slides with PBS 3 times for 2 minutes each. Incubate slides with serum blocking solution (SL038, Solarbio, China) for $30 \mathrm{~min}$. Incubate with Osteocalcin primary antibody $(1: 200$, ab13420, Abcam, USA) at $4{ }^{\circ} \mathrm{C}$ overnight. Wash and incubate with biotinylated second antibody (1:2,000, ab205719, Abcam, USA). Wash and incubate with streptavidin-enzyme conjugate.

\section{Statistical analysis}

One-way ANOVA was used to analyze the significance of differences among groups. A P value of less than 0.05 was considered significant. SPSS 16.0 software (SPSS Inc., Chicago, IL, USA) was used for analysis.

\section{Results}

\section{Characterization of Wnt4-DPSCs-IPs}

Five surface markers were chosen to characterize Wnt4DPSCs-IPs. STRO-1, CD90 and CD105 were selected as positive markers to characterize mesenchymal stem cells, hematopoietic CD34 and CD45 were chosen as negative markers. As shown in Figure 1, positive reaction was displayed by $89.93 \%$ of Wnt4-DPSCs-IPs for STRO-1, $85.89 \%$ for CD90 and $77.36 \%$ for CD105. While negative reaction was found for CD34 and CD45. The results suggested that Wnt4 overexpression did not change stem cell characteristics of DPSCs-IPs.

\section{Multi-differentiation potential of Wnt4-DPSCs-IPs}

We further tested the multi-differentiation potential of Wnt4-DPSCs-IPs. As we expected, Wnt4-DPSCs-IPs could be positively stained by Alizarin red, oil-red $\mathrm{O}$ and toluidine blue (Figure 2). Wnt4-DPSCs-IPs still retain the potential of multidirectional differentiation.

\section{Characterization of Wnt4-DPSCs-IPs/PHBV}

We first measured cell proliferation of Wnt4-DPSCsIPs on PHBV. MTT showed that no significant difference was detected on OD value between Wnt4-DPSCs-IPs and Wnt4-DPSCs-IPs/PHBV at 1, 3 and 7 days (Figure $3 A$ ). PHBV did not affect cell proliferation of Wnt4-DPSCsIPs. Next, ALP activity was tested with Wnt4-DPSCs-IPs and Wnt4-DPSCs-IPs/PHBV. No significant difference was shown (Figure 3B). Last, we performed scanning electron microscope with Wnt4-DPSCs-IPs/PHBV. In Figure $3 C$, we may find the rough surface of the PHBV. A large number of porous structures were detected as well as the cell attachment.

\section{Wnt4-DPSCs-IPs in bone regeneration in rats}

Micro-CT and histopathology were performed. Figure $4 A$ showed that the PHBV group demonstrated certain bone repair; while DPSCs-IPs/PHBV has less bone repair capacity than DPSCs-NPs/PHBV, but better capacity than PHBV; Wnt4-DPSCs-IPs/PHBV showed the improved bone repair potential which was close to DPSCs-NPs/ PHBV. Bone mineral density (BMD) and bone volume over total volume (BV/TV) were analyzed to show the bone repair potential of each group. Micro-CT analysis showed that BMD and BV/TV were decreased in DPSCsIPs/PHBV compared with DPSCs-NPs/PHBV and were restored in Wnt4-DPSCs-IPs/PHBV compared with DPSCs-IPs/PHBV (Figure 4B). The immunohistochemistry results showed that osteocalcin expressed at a higher level in DPSCs-NPs/PHBV as well as in Wnt4-DPSCs-IPs/PHBV, 

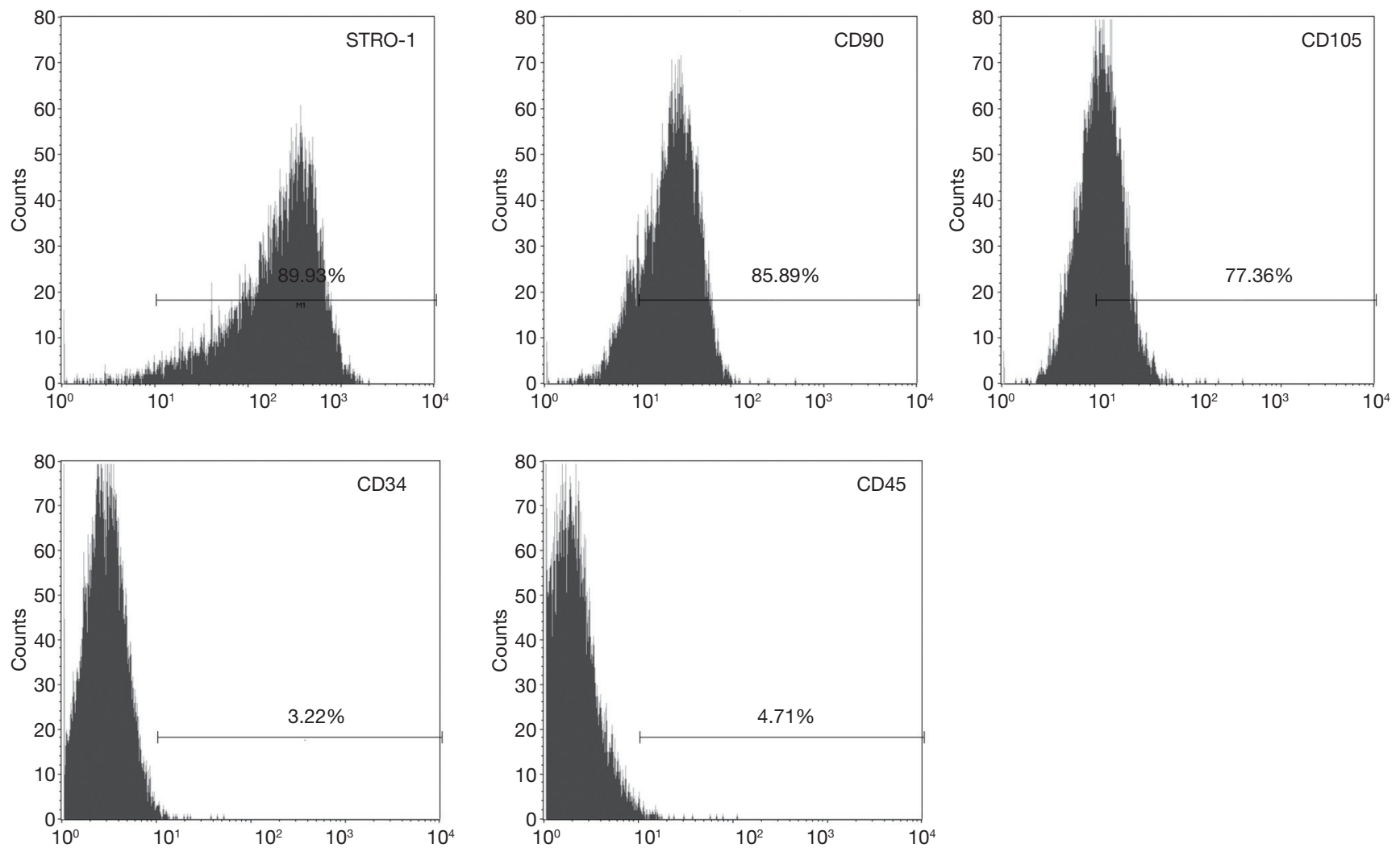

Figure 1 Characterization of Wnt4-DPSCs-IPs. Flow cytometry for characterization of Wnt4-DPSCs-IPs. Expression of surface markers STRO-1, CD90, CD105, CD34 and CD45 were detected. DPSCs-IPs, inflammatory dental pulp stem cells.

while a lower level in PHBV and DPSCs-IPs/PHBV, suggested that new bone formation were more active in both DPSCs-NPs/PHBV and Wnt4-DPSCs-IPs/PHBV (Figure 4C).

\section{Discussion}

In the present study, a possible strategy to improve the impaired osteogenic potential of DPSCs-IPs was tested, the feasibility and effectiveness were verified in vivo. Wnt4 restoration promoted the osteogenic differentiation of DPSCs-IPs. Wnt4-DPSCs-IPs/PHBV showed developed ability of reconstructing bone defect in rats.

Inflammatory dental pulp tissue-derived stem cells have broadened the source of mesenchymal stem cells. They have great potential in the application since no damage to normal tissues was needed any more (20,21). However, the only problem lies in the decrease of osteogenic capacity. Therefore, finding key factors to improve osteogenic ability of DPSCs-IPs can fundamentally solve the current problems. Wnt4 has been shown to inhibit bone resorption and its expression was also down regulated in the DPSCsIPs. The expression of Wnt4 is closely related to several stem cell osteogenic markers. We believe that Wnt4 is a key factor in increasing osteogenic potential of DPSCs-IPs.

Next, we investigated whether restoring Wnt4 expression would affect the stem cell characteristics of DPSCs-IPs. We found that Wnt4 overexpression would not change the characteristics of DPSCs-IPs as stem cells. In addition, Wnt4DPSCs-IPs still retained multidirectional differentiation potential, especially osteogenic differentiation, which has laid a good foundation for its future clinical application.

The effect of PHBV on the proliferation and osteogenic differentiation of Wnt4-DPSCs-IPs also needs to be elucidated before in vivo studies $(22,23)$. Our results showed that after loading Wnt4-DPSCs-IPs on PHBV, the proliferation and osteogenic differentiation ability of the complexes were not statistically different from Wnt4-DPSCs-IPs, which suggested that we may use the complexes in vivo to repair bone defect. 
Alizarin red staining
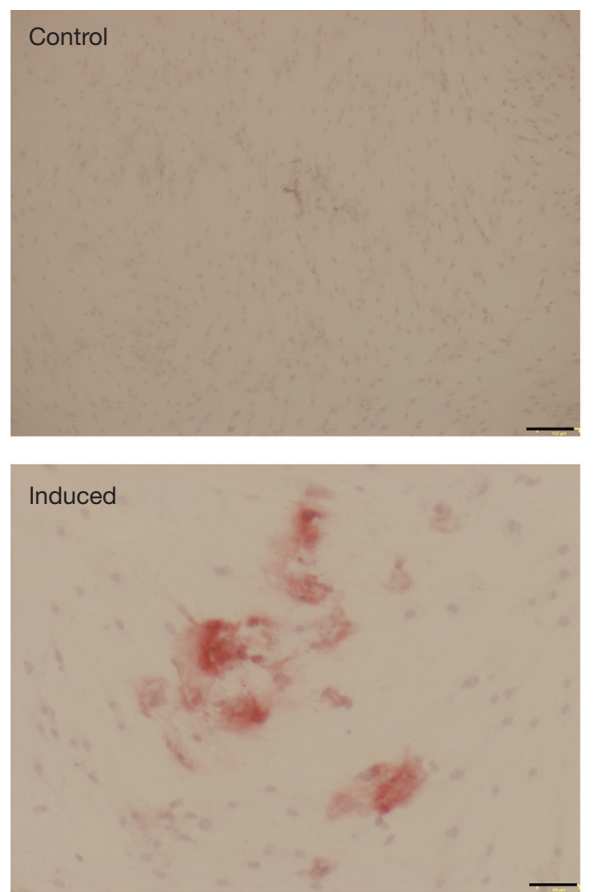

Oil-red O staining
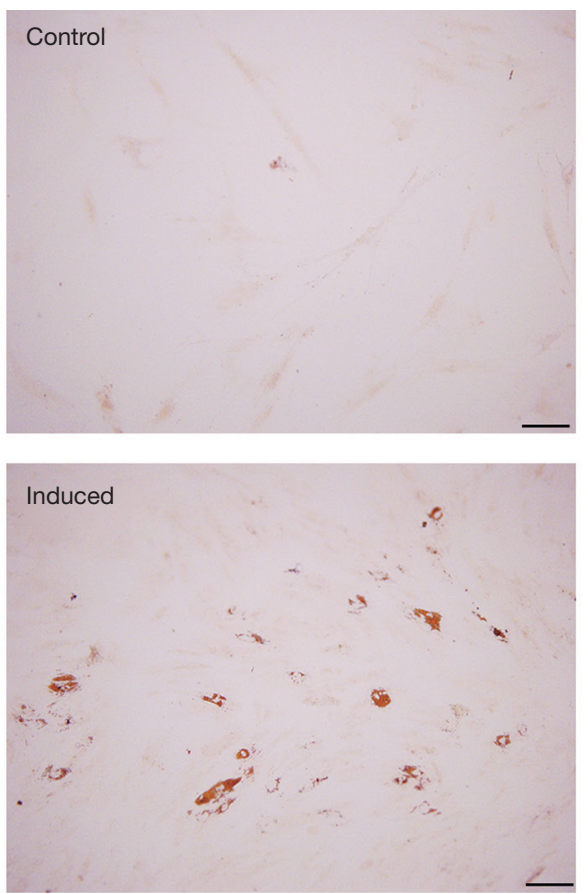

Toluidine blue
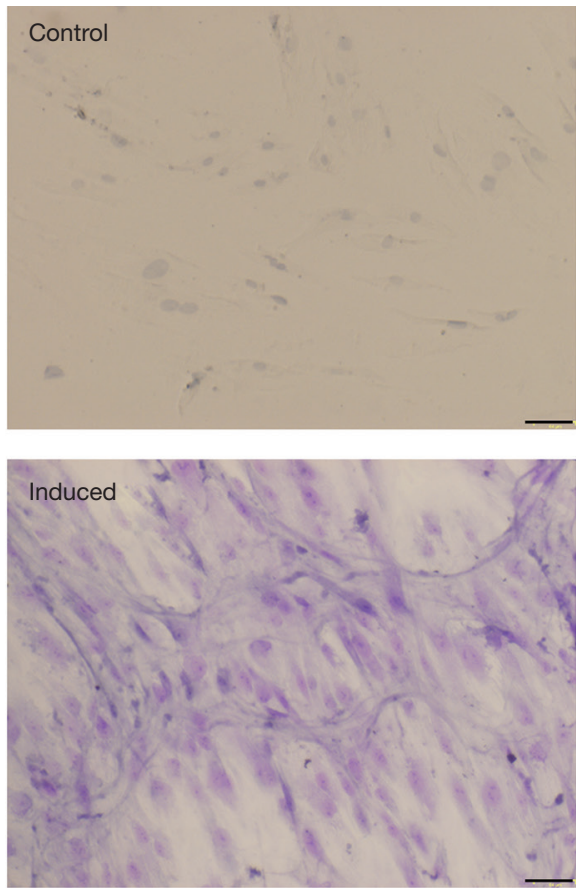

Figure 2 Multi-differentiation potential of Wnt4-DPSCs-IPs. Two weeks after induction of multidirectional differentiation, Alizarin red, oilred $\mathrm{O}$ and toluidine blue were stained with Wnt4-DPSCs-IPs. Scale bars $=100 \mu \mathrm{m}$. DPSCs-IPs, inflammatory dental pulp stem cells.

A

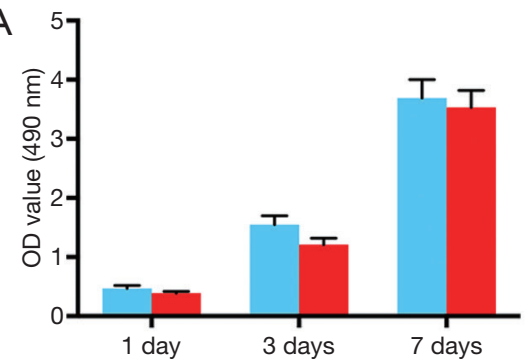

C

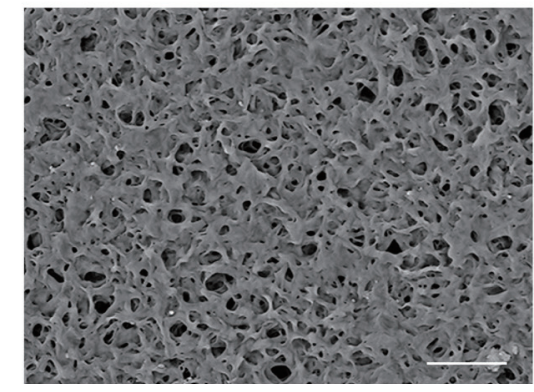

B
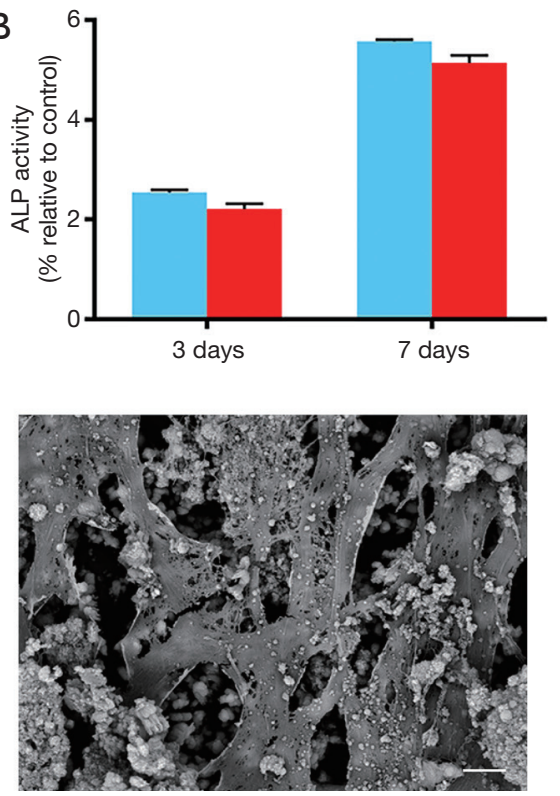

Wnt4-DPSCs-IPs

Wnt4-DPSCs-IPs/PHBV

Figure 3 Characterization of Wnt4-DPSCs-IPs/PHBV. (A) Wnt4-DPSCs-IPs proliferation on PHBV; (B) osteogenic potential of Wnt4DPSCs-IPs on PHBV; (C) scanning electron microscopy of Wnt4-DPSCs-IPs/PHBV. Scale bars $=1 \mu \mathrm{m}$. DPSCs-IPs, inflammatory dental pulp stem cells; PHBV, poly (hydroxybutyrate-co-valerate). 

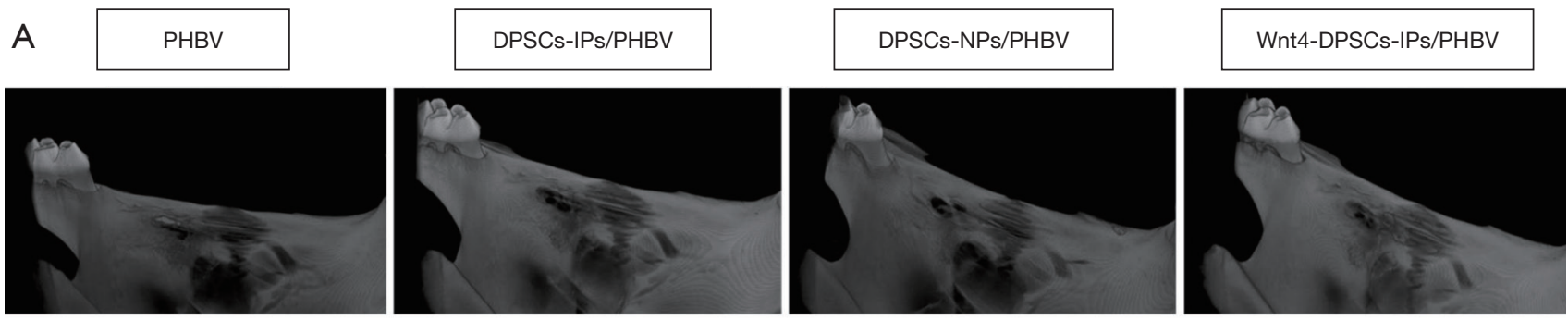

B
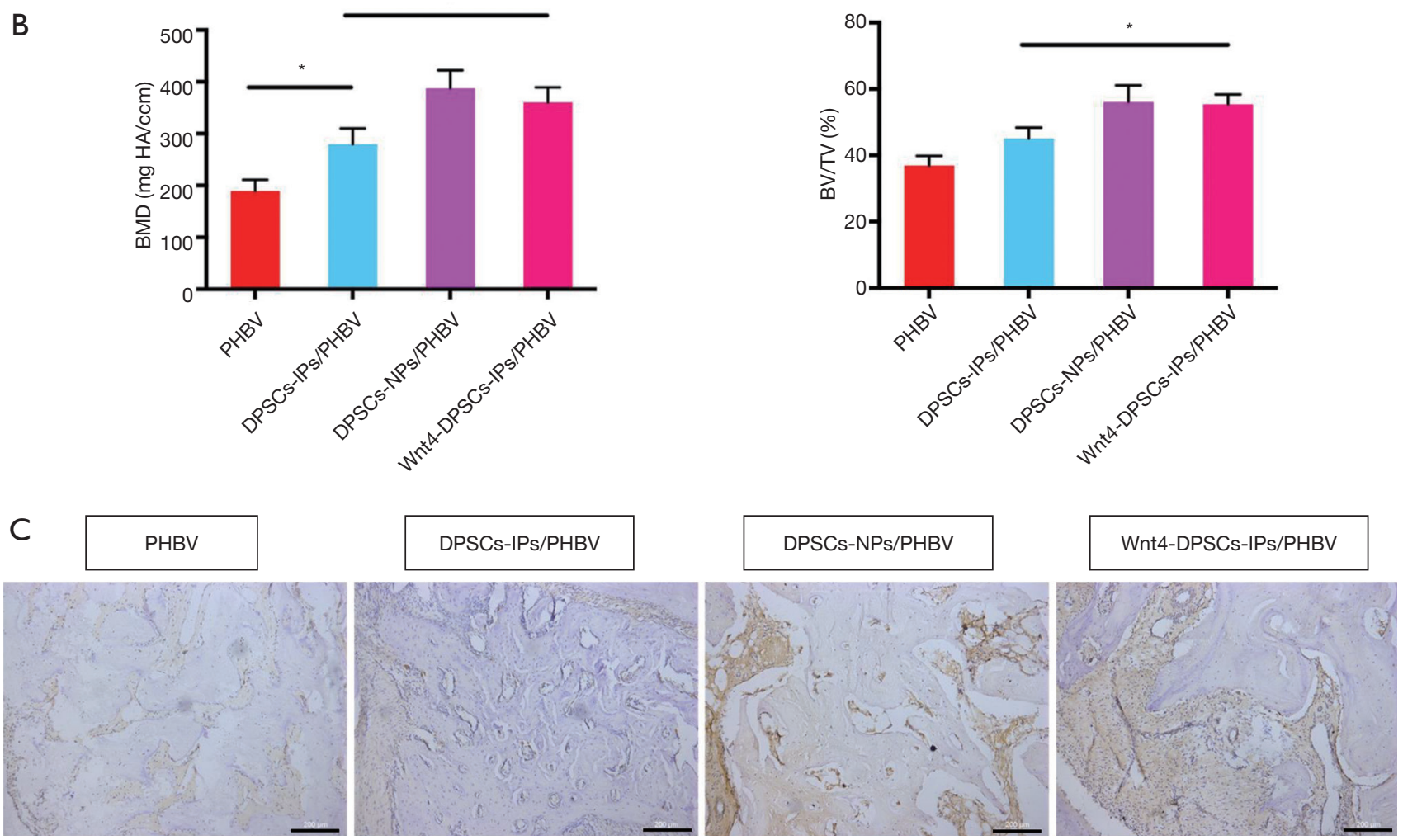

Figure 4 Wnt4-DPSCs-IPs in bone regeneration in rats. (A) Micro-CT images of bone defects 12 weeks after transplantation. Bone defects are clearly marked; (B) BMD and BV/TV were calculated; (C) osteocalcin expression in new bone formation area. Scale bars =200 $\mu \mathrm{m}$. *, $\mathrm{P}<0.05$. DPSCs-IPs, inflammatory dental pulp stem cells; DPSCs-NPs, normal dental pulp stem cells; PHBV, poly (hydroxybutyrate-covalerate); BMD, bone mineral density; BV/TV, bone volume over total volume.

The results of in vivo experiments showed that the PHBV group had no bad influence on bone repair, proving that PHBV is biologically safety in vivo and may be used in tissue regeneration in the clinic. While DPSCs-IPs/ PHBV, DPSCs-NPs/PHBV and Wnt4-DPSCs-IPs/PHBV have much better effect. DPSCs-IPs/PHBV has a slightly decreased bone repair effect than DPSCs-NPs/PHBV, since the osteogenic capacity was inhibited in inflammatory environment. Wnt4 restoration improved the osteogenic potential of DPSCs-IPs in vivo.

\section{Acknowledgments}

Funding: Shaanxi Province Science and Technology Coordinated Innovation Project (No. 2016KTCL03-13).

\section{Footnote}

Reporting Checklist: The authors have completed the ARRIVE reporting checklist. Available at http://dx.doi. org/10.21037/apm-20-460 
Data Sharing Statement: Available at http://dx.doi. org/10.21037/apm-20-460

Peer Review File: Available at http://dx.doi.org/10.21037/ apm-20-460

Conflicts of Interest: All authors have completed the ICMJE uniform disclosure form (available at http://dx.doi. org/10.21037/apm-20-460). The authors have no conflicts of interest to declare.

Ethical Statement: The authors are accountable for all aspects of the work in ensuring that questions related to the accuracy or integrity of any part of the work are appropriately investigated and resolved. All animals are treated in accordance with the guidelines of Xi'an Jiaotong University College of Medicine for Experimental Animals. All procedures performed were in accordance with the ethical standards of Xi'an Jiaotong University College of Medicine for Experimental Animals. The present study does not contain human participants.

Open Access Statement: This is an Open Access article distributed in accordance with the Creative Commons Attribution-NonCommercial-NoDerivs 4.0 International License (CC BY-NC-ND 4.0), which permits the noncommercial replication and distribution of the article with the strict proviso that no changes or edits are made and the original work is properly cited (including links to both the formal publication through the relevant DOI and the license). See: https://creativecommons.org/licenses/by-nc-nd/4.0/.

\section{References}

1. Li Y, Nan X, Zhong TY, et al. Treatment of periodontal bone defects with stem cells from inflammatory dental pulp tissues in miniature swine. Tissue Eng Regen Med 2019;16:191-200.

2. Alongi DJ, Yamaza T, Song Y, et al. Stem/progenitor cells from inflamed human dental pulp retain tissue regeneration potential. Regen Med 2010;5:617-31.

3. Li Y, Zhao S, Nan X, et al. Repair of human periodontal bone defects by autologous grafting stem cells derived from inflammatory dental pulp tissues. Stem Cell Res Ther 2016;7:141.

4. Boyle M, Chun C, Strojny C, et al. Chronic inflammation and angiogenic signaling axis impairs differentiation of dental-pulp stem cells. PLoS One 2014;9:e113419.
5. Al-Habib M, Huang GT. Dental mesenchymal stem cells: dental pulp stem cells, periodontal ligament stem cells, apical papilla stem cells, and primary teeth stem cells-isolation, characterization, and expansion for tissue engineering. Methods Mol Biol 2019;1922:59-76.

6. Cristaldi M, Mauceri R, Tomasello L, et al. Dental pulp stem cells for bone tissue engineering: a review of the current literature and a look to the future. Regen Med 2018. [Epub ahead of print].

7. Di Benedetto A, Carbone C, Mori G. Dental pulp stem cells isolation and osteogenic differentiation: a good promise for tissue engineering. Methods Mol Biol 2014;1210:117-30.

8. Dong Q, Wang Y, Mohabatpour F, et al. Dental pulp stem cells: isolation, characterization, expansion, and odontoblast differentiation for tissue engineering. Methods Mol Biol 2019;1922:91-101.

9. Kaneko T, Gu B, Sone PP, et al. Dental pulp tissue engineering using mesenchymal stem cells: a review with a protocol. Stem Cell Rev Rep 2018;14:668-76.

10. Leyendecker Junior A, Gomes Pinheiro CC, Lazzaretti Fernandes T, et al. The use of human dental pulp stem cells for in vivo bone tissue engineering: a systematic review. J Tissue Eng 2018;9:2041731417752766.

11. Huang AH, Chen YK, Lin LM, et al. Isolation and characterization of dental pulp stem cells from a supernumerary tooth. J Oral Pathol Med 2008;37:571-4.

12. Karaöz E, Doğan BN, Aksoy A, et al. Isolation and in vitro characterisation of dental pulp stem cells from natal teeth. Histochem Cell Biol 2010;133:95-112.

13. Kerkis I, Kerkis A, Dozortsev D, et al. Isolation and characterization of a population of immature dental pulp stem cells expressing OCT-4 and other embryonic stem cell markers. Cells Tissues Organs 2006;184:105-16.

14. Gregory CA, Gunn WG, Reyes E, et al. How Wnt signaling affects bone repair by mesenchymal stem cells from the bone marrow. Ann N Y Acad Sci 2005;1049:97-106.

15. Yu B, Chang J, Liu Y, et al. Wnt4 signaling prevents skeletal aging and inflammation by inhibiting nuclear factor-kappaB. Nat Med 2014;20:1009-17.

16. Yu B, Chang J, Liu Y, et al. Addendum: Wnt4 signaling prevents skeletal aging and inflammation by inhibiting nuclear factor-kappaB. Nat Med 2015;21:1101.

17. Van Camp JK, Beckers S, Zegers D, et al. Wnt signaling and the control of human stem cell fate. Stem Cell Rev Rep 2014;10:207-29.

18. Chang J, Sonoyama W, Wang Z, et al. Noncanonical Wnt4 signaling enhances bone regeneration of mesenchymal 
stem cells in craniofacial defects through activation of p38 MAPK. J Biol Chem 2007;282:30938-48.

19. Zhong TY, Zhang ZC, Gao YN, et al. Loss of Wnt4 expression inhibits the odontogenic potential of dental pulp stem cells through JNK signaling in pulpitis. Am J Transl Res 2019;11:1819-26.

20. Fawzy El-Sayed KM, Elsalawy R, Ibrahim N, et al. The dental pulp stem/progenitor cells-mediated inflammatoryregenerative axis. Tissue Eng Part B Rev 2019;25:445-60.

21. Sloan AJ, Smith AJ. Stem cells and the dental pulp:

Cite this article as: Zhong T, Gao Y, Qiao H, Zhou H, Liu Y. Elevated osteogenic potential of stem cells from inflammatory dental pulp tissues by Wnt4 overexpression for treating bone defect in rats. Ann Palliat Med 2020;9(5):2962-2969. doi: 10.21037/ apm-20-460 potential roles in dentine regeneration and repair. Oral Dis 2007;13:151-7.

22. Kim SS, Sun Park M, Jeon O, et al. Poly(lactide-coglycolide)/hydroxyapatite composite scaffolds for bone tissue engineering. Biomaterials 2006;27:1399-409.

23. Zhao T, Jing Y, Zhou X, et al. PHBV/PLA/Colbased nanofibrous scaffolds promote recovery of locomotor function by decreasing reactive astrogliosis in a hemisection spinal cord injury rat model. J Biomed Nanotechnol 2018;14:1921-33. 